\title{
Symptoms in patients with diverticular disease should not be labelled as IBS
}

\author{
Charlotte Louise Kvasnovsky • Savvas Papagrigoriadis
}

Accepted: 29 November 2014 / Published online: 10 December 2014

(C) Springer-Verlag Berlin Heidelberg 2014

\section{Dear Editor:}

We read with great interest the article 'Incidence and predictive factors of irritable bowel syndrome after acute diverticulitis in Korea' published in October 2014 [1]. Upon reading it, we came to the conclusion that these patients were suffering from symptomatic diverticular disease following an attack of diverticulitis, not irritable bowel syndrome (IBS).

The authors retrospectively identified patients admitted with diverticulitis and then conducted a telephone survey to assess symptoms post-diverticulitis. Of the patients, $12.8 \%$ met criteria for IBS, although unfortunately they do not further detail patients' post-discharge symptoms. Recurrent abdominal pain and change in stool consistency and/or pattern would be consistent with a definition of diverticular disease, a known syndrome following acute diverticulitis [2]. Remarkably, the authors do not identify a single patient with diverticular disease within their cohort.

The pedantic point is that as a functional disorder, IBS cannot, by definition, have a structural deformity in the colon. As such, patients suffering from IBS-like symptoms who have colonic diverticula have diverticular disease. However, this is an issue with the definition of IBS (which should certainly include patients with asymptomatic diverticulosis and classic IBS pain), and to belabour it undermines the substantive issue in the manuscript by Jung et al.

There is some evidence to suggest left lower quadrant pain may be the most specific symptom of diverticular disease, but this was not asked [3]. Additionally, patients with diverticular disease may have elevated levels of faecal calprotectin as compared to patients with IBS [4]. However, without these additional details, any persistent pain following diverticulitis should be diagnosed as diverticular disease.

C. L. Kvasnovsky $(\bowtie) \cdot$ S. Papagrigoriadis

King's College Hospital, London, UK

e-mail: ckvasno@gmail.com
We further suggest that the Cox regression model (Figure 2) is unable to demonstrate time to symptoms following diverticulitis as described [1]. The researchers carried out follow-up only at one time point, in July 2013, on patients hospitalized between 2007 and 2012. They were thus unable to identify when patients developed abdominal symptoms following their episode of diverticulitis. (Indeed, we would imagine that those patients labelled here with IBS likely had persistent symptoms of diverticular disease following hospitalization.) Thus, the length of time to 'development of IBS' in their model is dependent only on the patients' initial date of hospitalization, not on any disease process.

In conclusion, these researchers found that $12.8 \%$ of patients admitted with acute diverticulitis had persistent symptoms of diverticular disease at follow-up, anywhere from 1 to 5 years following hospitalization. Following acute diverticulitis, patients are at risk of persistent symptoms and should be followed up after discharge.

\section{References}

1. Jung S, Lee H, Chung $H$ et al (2014) Incidence and predictive factors of irritable bowel syndrome after acute diverticulitis in Korea. Int $\mathrm{J}$ Color Dis 29(11):1369-1376

2. Simpson J, Neal KR, Scholefield JH, Spiller RC (2003) Patterns of pain in diverticular disease and the influence of acute diverticulitis. Eur J Gastroenterol Hepatol 15(9):1005-1010

3. Tursi A, Elisei W, Picchio M, Giorgetti GM, Brandimarte G (2014) Moderate to severe and prolonged left lower-abdominal pain is the best symptom characterizing symptomatic uncomplicated diverticular disease of the colon: a comparison with fecal calprotectin in clinical setting. J Clin Gastroenterol. doi:10.1097/MCG.0000000000000094

4. Tursi A, Brandimarte G, Elisei W, Giorgetti GM, Inchingolo CD, Aiello F (2009) Faecal calprotectin in colonic diverticular disease: a case-control study. Int J Color Dis 24(1):49-55 